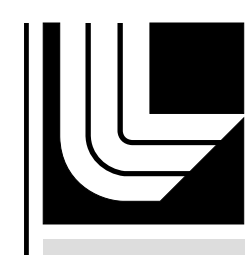

LAWRENCE LIVERMORE N A T IO N A L LABORATORY

\title{
Coupling Magnetic Fields and ALE Hydrodynamics for 3D Simulations of MFCG's
}

Daniel White, Robert Rieben, Brad Wallin

October 7, 2006

2006 IEEE International Conference on Megagauss Magnetic Fields and Realted Topics 
This document was prepared as an account of work sponsored by an agency of the United States government. Neither the United States government nor Lawrence Livermore National Security, LLC, nor any of their employees makes any warranty, expressed or implied, or assumes any legal liability or responsibility for the accuracy, completeness, or usefulness of any information, apparatus, product, or process disclosed, or represents that its use would not infringe privately owned rights. Reference herein to any specific commercial product, process, or service by trade name, trademark, manufacturer, or otherwise does not necessarily constitute or imply its endorsement, recommendation, or favoring by the United States government or Lawrence Livermore National Security, LLC. The views and opinions of authors expressed herein do not necessarily state or reflect those of the United States government or Lawrence Livermore National Security, LLC, and shall not be used for advertising or product endorsement purposes. 


\title{
Coupling Magnetic Fields and ALE Hydrodynamics for 3D Simulations of MFCGs
}

\author{
Daniel A. White, Robert N. Rieben and Brad K. Wallin \\ Lawrence Livermore National Laboratory, Livermore, CA, USA
}

\begin{abstract}
We review the development of a full 3D multiphysics code for the simulation of explosively driven Magnetic Flux Compression Generators (MFCG) and related pulse power devices. In a typical MFCG the device is seeded with an initial electric current and the device is then detonated. The detonation compresses the magnetic field and amplifies the current. This is a multiphysics problem in that detonation kinetics, electromagnetic diffusion and induction, material deformation, and thermal effects are all important. This is a tightly coupled problem in that the different physical quantities have comparable spatial and temporal variation, and hence should be solved simultaneously on the same computational mesh.
\end{abstract}

\section{INTROUDCTION}

Our approach is to begin with the LLNL Arbitrary Lagrangian-Eulerian code ALE3D. ALE3D is a parallel, 3D, unstructured-grid finite element code that has support for structural mechanics, hydrodynamics, energetic materials, and heat transfer. The hydrodynamics algorithms used in ALE3D are standard and can be found in Benson ${ }^{1}$ and hence will not be discussed further. The new electromagnetics module is based on a compatible discretization (fields on mesh edges, fluxes on mesh faces) of Maxwell's equations with the displacement current term neglected, resulting in a dynamo equation. This discretization of the dynamo equation satisfies the divergence-free nature of the magnetic fields without need of any Lagrange constraint, penalty term, or "divergence cleaning" procedure. The dynamo equation consists of a diffusion term and an advection term; these two terms are treated in an operator split manner.

Due to the compatible discretization of the dynamo equation the numerical solution in the Lagrangian frame is straightforward, the advection term simply disappears. However, as the mesh becomes distorted it is necessary to switch to an ALE method, which means that material now flows through the mesh. The ALE method is implemented as a Lagrange step followed by a remap step; this remap step is equivalent to advection with a nonphysical mesh relaxation velocity. Accurate advection has been a long-standing issue with ALE methods and is particularly difficult for electromagnetics due to the divergence-free nature of the fields. We have developed a new advection method for 3D unstructured grids that satisfies the divergence-free condition and is second-order accurate for smooth fields. A custom monotonic flux-limiting algorithm was also developed; this algorithm is automatically applied on shock fronts.

\section{Dynamo Equation}

We employ an E-B field formulation of the electromagnetics. For problems without material motion the two key equations are 


$$
\begin{aligned}
& \sigma E=\nabla \times \frac{1}{\mu} B+\sigma \nabla \phi-J_{s} \\
& \frac{\partial B}{\partial t}=-\nabla \times E
\end{aligned}
$$

where $\mathrm{E}$ is the electric field, $\mathrm{B}$ is the magnetic flux density, $\sigma$ is the electrical conductivity, and $\mu$ is the magnetic permeability. The current $J_{s}$ is an independent current source which may or may not exist depending upon the problem, likewise $\phi$ is scalar electric potential that may or may not exist. It is assumed that E or B is specified on the external boundary. These two equations are discretized using a mixed finite element method using $H$ (curl) "edge" basis functions for the E-field and $H($ div ) "face" basis functions for the B-field. The advantage of this formulation is that is it provably stable, it allows for jump discontinuity of $\mathrm{E}$ and $\mathrm{B}$ across materials interfaces, and the divergence conditions

$$
\nabla \bullet B=0 \quad \nabla \bullet \sigma E=0
$$

are satisfied exactly without the need for any additional constraint or penalty ${ }^{2}$. These equations are integrated in time using an accurate implicit method. This formulation has been extensively verified and has been shown to be second order accurate in both space and time ${ }^{2}$.

When dealing with moving materials the electromagnetic equations can be expressed in either the laboratory frame (Eulerian method) or the material frame (Lagrangian method). Let $x$ denote the label of a point in the laboratory where the motion takes place, and let $X$ denote the label of a point in the material. There exists a time-dependent bijective mapping relating these two labels of the same point,

$$
x=x(X, t) \quad X=X(x, t)
$$

Let a vector field defined with respect to the laboratory frame be denoted with a prime, e.g. $F^{\prime}(x, t)$ and the same vector field defined with respect to the material frame be unprimed, e.g. $F(X, t)$. Voltage and flux in these two different frames of references are related by

$$
\begin{array}{ccc}
\text { Material } & \text { Laboratory } \\
E \bullet d x & = & \left(E^{\prime}+v^{\prime} \times B^{\prime}\right) \bullet d x^{\prime} \\
B \bullet d a & = & B^{\prime} \bullet d a^{\prime}
\end{array}
$$

where $d x=J^{T} d x^{\prime} \quad d a=|J| J^{-1} d a^{\prime} \quad J_{i j}=\partial X_{j} / \partial x_{i}$. For theoretical analysis it is convenient to express the electromagnetics in the form of the dynamo equation in the laboratory frame

$$
\frac{\partial B^{\prime}}{\partial t}=-\nabla \times \frac{1}{\sigma \mu} \nabla \times B^{\prime}+\nabla \times v^{\prime} \times B^{\prime}
$$

where the source terms have been neglected for clarity. The first term on the right is diffusion, the second term is advection, and the ration of these is the magnetic Reynolds number $M R=\frac{\nu L}{\lambda} \quad \lambda=\frac{1}{\sigma \mu}$ where $\mathrm{L}$ is the characteristic size and $\lambda$ is the magnetic diffusivity. For problems in which the diffusion and advection terms are approximately equal and opposite, we have near equilibrium and time integration of the dynamo equation requires special care. But for problems in which either diffusion or advection 
dominate it is acceptable to employ an operator splitting of the equations. In operator splitting of the dynamo equations the sequence is: 1) advect the B field without diffusion, 2) diffuse the B field without advection.

In the material frame the dynamo equation is

$$
\frac{\partial B}{\partial t}=\nabla \times \frac{1}{\sigma \mu} \nabla \times B
$$

where it is understood that the curl operator is with respect to the material coordinates. For the special case of a perfectly conducting material this gives $\frac{\partial B}{\partial t}=0$, the frozen-influx theorem. The operator splitting of the dynamo equation is particularly simple in the material frame: 1) move the mesh nodes according to the $\mathrm{JxB}$ forces while maintaining $\frac{\partial B}{\partial t}=0$ during mesh motion, 2) diffusion of the B-field. It is essential to note that the transformations $d x=J^{T} d x^{\prime} \quad d a=|J| J^{-1} d a^{\prime}$ are built-in to the $H$ (curl) and $H$ (div) finite element basis functions, respectively. Therefore $\frac{\partial B}{\partial t}=0$ is equivalent to simply keeping the magnetic degrees-of-freedom, which are the magnetic flux through each mesh face, constant.

\section{Electromagnetic and Hydrodynamic Coupling}

In the material frame the equation of motion for a solid is

$$
\rho \frac{\partial^{2} u}{\partial t^{2}}=\nabla \bullet \vec{S}+F
$$

where $\rho$ is the material mass density, $\mathrm{u}$ is the displacement vector, $\mathrm{S}$ is the Cauchy stress tensor, and $\mathrm{F}$ is an independent volumetric body force. There are two different approaches for coupling the electromagnetic forces to the hydrodynamics. The first approach is to simply compute $J x B$ in every mesh element and add this to the body force F. The second approach is to compute the Maxwell stress tensor $T_{i j}=\frac{1}{\mu}\left(B_{i} B_{j}-\frac{1}{2} \delta_{i j} B_{k} B_{k}\right)$ and add this to the Cauchy stress tensor. Both options are implemented in ALE3D.

\section{Magnetic Advection}

The ALE3D code performs an optional equipotential relaxation of the mesh. This is important for problems with gross deformation of the mesh, it prevents the mesh from becoming tangled. If relaxation is performed, fields defined on the "old" mesh must be remapped to the "new" mesh. This is equivalent to Eulerian advection, but with a fictitious mesh velocity $v_{m}$. It is interesting to note that it is possible to implement a pure Eulerian formulation as a Lagrange step followed by a complete remap step in which the mesh snaps back to its original configuration at every step, and this is an option within ALE3D.

The goal of magnetic advection is to update the B-field according to

$$
\frac{\partial B}{\partial t}=-\nabla \times v_{m} \times B
$$

where $v_{m}$ is the mesh velocity. Since the magnetic degrees-of-freedom are the flux 
through each mesh face, integrating this equation over a mesh face and using central difference time integration yields

$$
\Phi^{\text {new }}=\Phi^{\text {old }}-\oint_{C} u \times B \bullet d l
$$

for updating the degrees-of-freedom. This is the basic equation of the constrained transport method, when implemented correctly it preserves the zero divergence of the Bfield. In our implementation the contour integral is around an intermediate mesh face defined as the average of the "old" and "new" mesh faces, as shown in Figure 1 below. The contour location is precisely where the mesh velocity is known. The integrand requires a continuous B-field, therefore we employ a patch-recovery process (specifically a volume weighted-average, although other methods may be used as well) to construct the continuous B-field.
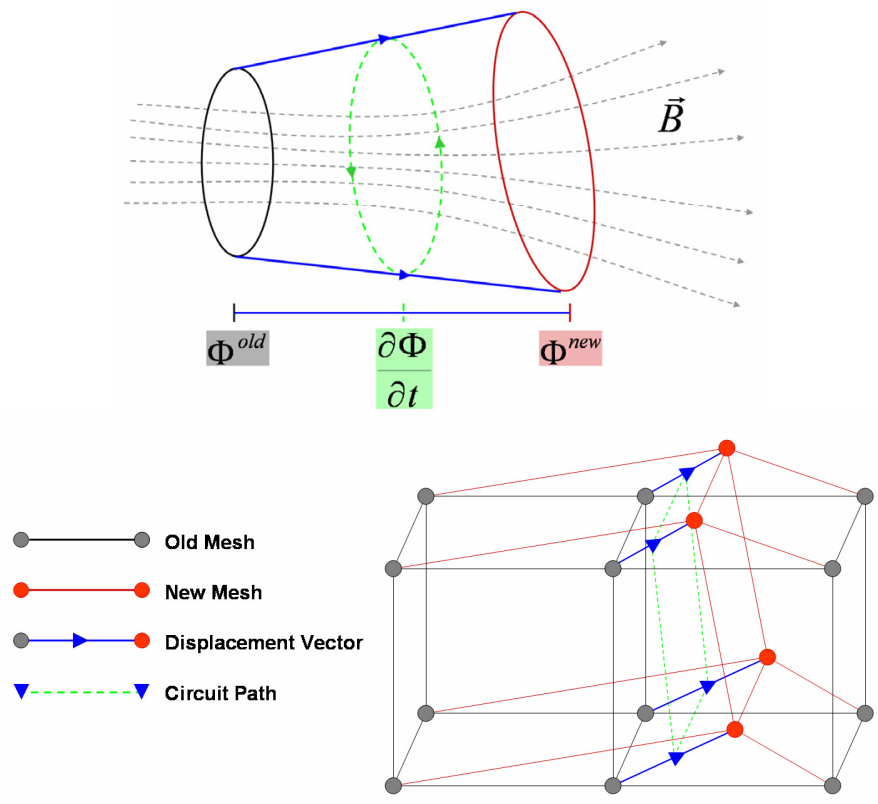

Figure 1. The top figure illustrates the constrained transport algorithm, which is in essence a central-difference discretization of Faraday's Law. The bottom figure shows how the intermediate face for the contour integral is constructed

We refer to the above algorithm as Algebraic Constrained Transport because it is valid for unstructured grids; it is a generalization of the original constrained transport algorithm that was developed for Cartesian grids ${ }^{4}$. The Algebraic Constrained Transport method is second-order accurate, it conserves divergence exactly, and it conserves magnetic field energy to $O\left(h^{2}\right)$. However, for strong shocks it generated "wiggles" in the B-field at the shock boundary, very similar to the classic Lax-Wendroff method for the 1D gas dynamics advection equation. To correct for this we apply an Algebraic Flux Correction ${ }^{3}$ step which guarantees a monotonic remap of the fields. The algorithm can only be summarized here. The basic steps are:

1) Compute the unlimited flux change using Algebraic Constrained Transport 
2) Loop over the entire mesh to detect all shock fronts

3) Apply flux limiting only to the mesh edges which lie on each shock front.

By construction this method is monotonic, and it still preserves the divergence free character of the B-field. However, much like other flux-limiting schemes used in gas dynamics, it effectively introduces a non-physical dissipation into the problem. Figure 2 shows how the method performs for a smooth field and for a shock, the flux limiting eliminates the "wiggles" but slightly rounds the corners of the shock.

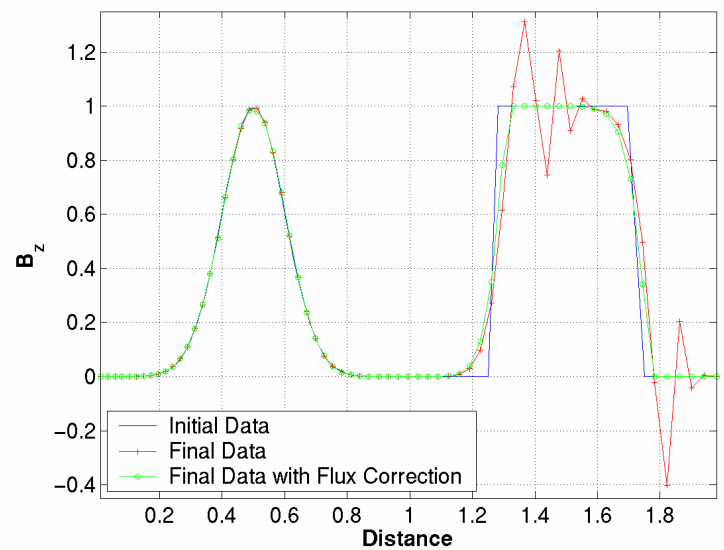

Figure 2. Evaluation of the combined Algebraic Constrained Transport - Algebraic Flux Correction for the B-field. This is a slice through a 3D mesh. In this simulation the Bfield has a Gaussian profile on the left, a square wave on the right, and the mesh is moving to the right. As can be seen the algorithm is very accurate for smooth fields, and is monotonic for shocks.

\section{RESULTS}

As mentioned in the introduction, simulation of explosive MFGC's is a challenging multiphysics problem. Clearly a full 3D simulation capability is required for helical generators, and it is useful for evaluation of perturbations or defects in coaxial generators as well. In this section we apply the ALE3D code to the Shearer et. al. "34" coaxial generator ${ }^{5}$. This generator has undoubtedly been simulated in great detail using $2 \mathrm{D}$ axissymmetric codes. Here we perform a full 3D simulation in order to verify the 3D algorithm described above. The coaxial generator is driven with a prescribed current, and the generator is simply short-circuited at the output. The primary output quantity is the total electric current, which compares quite well to the measured data. In addition, it is possible to investigate loss mechanisms as shown in Figure 5. This loss is due to heating diffusion of currents and fields into the conductors. 

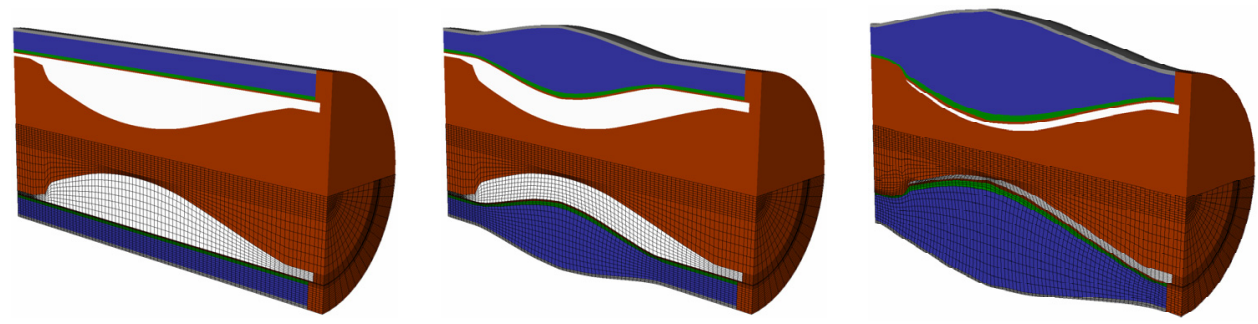

Figure 3. Material deformation in the Shearer et. al. "34" generator. This is a sequence of snapshots at $\mathrm{t}=0.0 \mu \mathrm{s}, \mathrm{t}=47.5 \mu \mathrm{s}$, and $\mathrm{t}=75 \mu \mathrm{s}$.
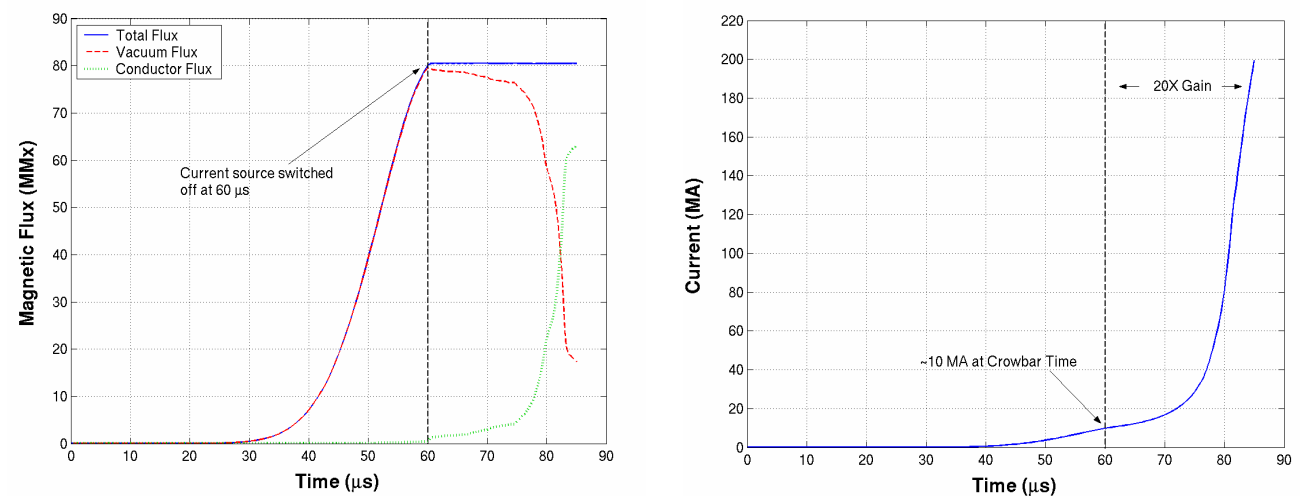

Figure 5. The left figure shows the magnetic flux as a function of time. The three lines are the total flux, the vacuum flux, and the conductor flux. The right figure is the total current vs. time, the gain agrees with the measured data ${ }^{5}$.

\section{ACKNOWLEDGMENT}

This work was performed under the auspices of the U.S. Department of Energy by University of California, Lawrence Livermore National Laboratory under Contract W7405-Eng-48. The project 04-ERD-86 was funded by the Laboratory Directed Research and Development Program.

\section{REFERENCES}

1. D. J. Benson, "Computational methods in Lagrangian and Eulerian hydrocodes," Comput. Methods Appl. Mech. Eng., vol. 99,1992, pp. 235-394.

2. R. Rieben, D. White, "Verification of high-order mixed finite element solution of transient magnetic diffusion problems," IEEE Trans. Magnetics, vol. 42, no. 1, 2006, pp. 25-39.

3. Rieben, R., N. et al., "An Arbitrary Lagrangian Eulerian discretization of MHD on 3D unstructured grids," LLNL report UCRL-JRNL-222113.

4. C. R. Evans, J. F. Hawley, 'Simulation of magnetohydrodynamic flows: a constrained transport method," The Astrophysical Journal, vol. 332, 1988, pp. 659-677.

5. J. W. Shearer, et. al., "Explosive driven magnetic field compression generators, " J. Appl. Physics, vol. 39, no. 4, 1968, pp.2102-2116. 